\title{
OPTOMECHANICAL TRANSDUCER-BASED SOFT AND HIGH FREQUENCY NANOSCALE CANTILEVER FOR ATOMIC FORCE MICROSCOPY
}

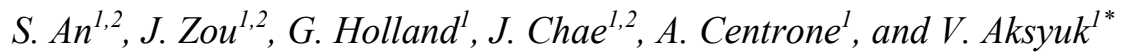

${ }^{1}$ Center for Nanoscale Science and Technology, National Institute of Standard and Technology, Gaithersburg, Maryland, USA

${ }^{2}$ Maryland Nanocenter, University of Maryland, College Park, Maryland, USA

\begin{abstract}
We introduce an optomechanical transducer-based nanoscale cantilever probe for atomic force microscopy (AFM). The high optical quality factor of the microdisk resonator enables detection of the nanoscale cantilever motion with high sensitivity. The low stiffness $(\approx 1 \mathrm{~N} / \mathrm{m})$ and high frequency (above $\approx 4 \mathrm{MHz}$ ) nanoscale cantilever provides both a wide bandwidth for fast motion detection and a high force sensitivity. We demonstrate the capabilities of the device in AFM for fast scanning (nominal $30 \mu \mathrm{m} \times 30 \mu \mathrm{m}, 39.06 \mathrm{~Hz}$ line rate, $2.93 \mathrm{~mm} / \mathrm{s}$ tip speed) with a fast settling time $(<2 \mu \mathrm{s})$. Furthermore, the detection of photo-thermal induced resonance (PTIR) signals at a $50 \mathrm{~nm}$ thick polymer film is also demonstrated.
\end{abstract}

\section{INTRODUCTION}

Fast-scanning AFM with sensitive force measurement is essential for investigating short time-constant motion or dynamics at small scales, and the demand for this capability is growing rapidly in various fields of nanoscale science and technology [1-3]. The key to realizing the desired performance is the use of a cantilever with a high mechanical resonance frequency $(\omega)$, in order to provide a wide detection bandwidth, and a low stiffness $(k)$, in order to provide high force sensitivity. These two goals can be realized by reducing the cantilever size to the nanoscale. However, if the cantilever size is reduced in this manner, optical transduction is inefficient for the typical beam bounce far-field or interferometric detection methods, used in conventional AFM systems. This occurs because the light cannot be focused only on the nanoscale cantilever due to the optical diffraction limit. For a given minimum size and mass $(m)$, the stiffness increases with larger $\omega$, since $k=m \omega^{2}$, resulting in low sensitivity.

Here we introduce a fully-integrated silicon microdisk cavity optomechanical transducer-based nanoscale cantilever to make a fast-scanning, sensitive AFM. An integrated near-field detection scheme [4] allows reduction of the mechanical probe cross-section to the $100 \mathrm{~nm}$ scale. A remarkable advantage of our cavity optomechanical transducer is its highly sensitive mechanical motion measurement, which is enabled by the strong interaction between photons that are confined inside the cavity and the cantilever motion. Optical shot noise, rather than thermal noise, fundamentally limits the motion readout precision, and optical power does not, in principle, have to be dissipated in the transducer. Readout precision is sufficient for the probe to operate at its fundamental thermo-mechanical limit. Among various types of optomechanical transducers such as suspended membranes [5], planar photonic crystal cavities [6], and microdisk resonators [7,8], we choose to utilize a microdisk type transducer for geometrical reasons: the cantilever can be simply incorporated around the rim of the microdisk, leading to an extended cantilever-disk interaction region, greatly enhancing the optomechanical coupling $g_{O M}$. This layout geometry facilitates the engineering of $\omega$ and $k$ through geometry variations, and enables practical experiments in AFM systems. Furthermore, the integrated chip (optomechanical transducer and waveguide) is connected by optical fiber, which eliminates the need for laser alignment and provides a stable output signal.

We use a custom adapter to incorporate our transducer chip in place of a conventional AFM cantilever chip into a commercial AFM and PTIR system, and demonstrate improved performance of both instruments.

\section{NANOFABRICATION OF THE DEVICE}

Figure 1 shows the nanofabrication process of the device. The devices are fabricated in a $260 \mathrm{~nm}$ thick silicon layer on top of the $1 \mu \mathrm{m}$ buried oxide (BOX) of a silicon-on-insulator (SOI) chip. Typically, multiple $5 \mathrm{~mm} \times 5 \mathrm{~mm}$ individual die are fabricated on a single $3 \mathrm{~cm} \times 3 \mathrm{~cm}$ chip. In this work electron (E)-beam and

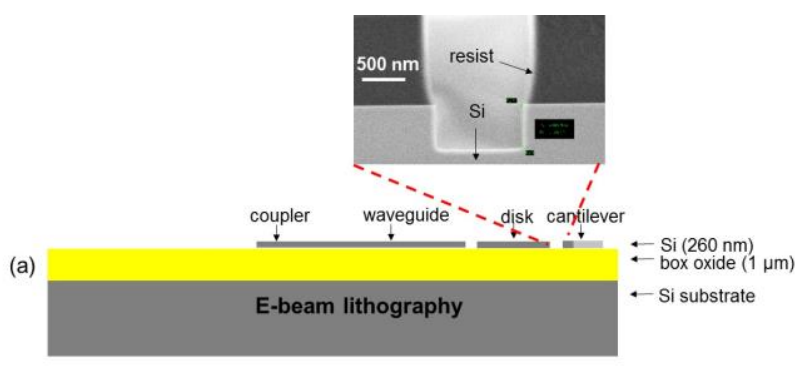

(b)

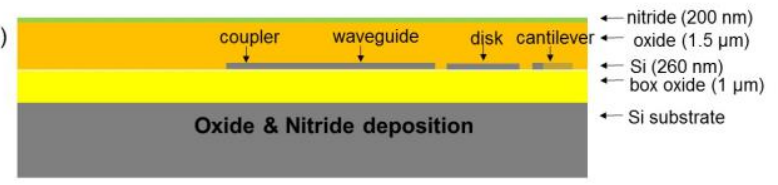

(c)

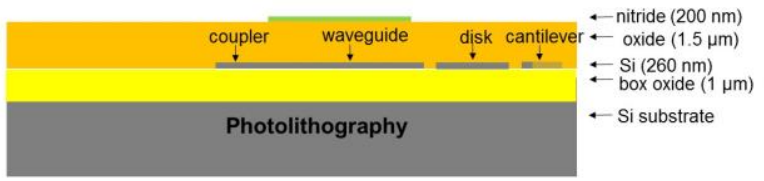

(d)

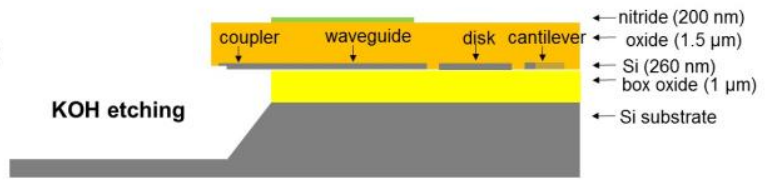

(e)

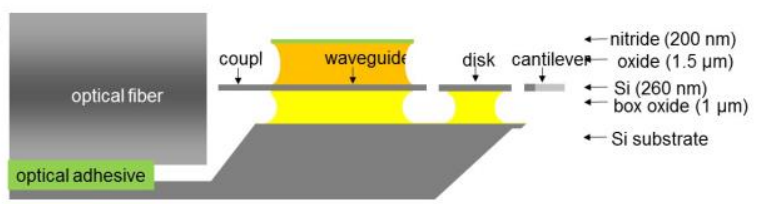

Figure 1: Nanofabrication of the optomechanical device. (a) E-beam lithography. (b) Oxide \& nitride depositions. (c) Photolithography and nitride dry etch (d) Oxide and $\mathrm{KOH}$ etching. (e) Cantilever releasing with wet HF etching, critical point drying, mechanical and FIB under-cut below the cantilever, and gluing the cleaved optical fiber. Inset shows sidewall profile from the Si etch, scale bar $500 \mathrm{~nm}$. 
multiple contact optical lithography steps are combined for defining device layers. This work is focused on device demonstration, and uses a few steps that are not compatible with batch fabrication. A much more sophisticated process aimed at batch fabrication of a wide class of similar devices is reported in a separate paper at this conference.

In the first step, E-beam lithography is used to define all features in the SOI Si, with critical features being the narrow gaps and the small cantilever width, followed by inductively-coupled plasma reactive ion etching for patterning the Si layer with a $\mathrm{C}_{4} \mathrm{~F}_{8}$ passivation-based $\mathrm{SF}_{6}$ etching technique [9] as shown in Fig. 1(a). Note that we check the etched vertical profile with a test silicon chip which has $1 \mu \mathrm{m}$ line pattern before device etching, as depicted in the inset of Fig. 1(a). We realize vertical and very smooth sidewall profiles, which are required for controlling optical mode shapes and achieving the high optical quality factor for high sensitivity and selectivity for in-plane mechanical motion.

Next step is the low-pressure chemical vapor deposition (LPCVD) of $\mathrm{SiO}_{2}$ and nitride as shown in Fig. 1(b). The oxide is used as optical cladding and sacrificial layer, and nitride as a mask layer for the subsequent Hydrofluoric acid (HF) release step. Nitride windows near the microdisk/cantilever and the fiber couplers are opened by photolithography and reactive ion etching, as shown in Fig. 1(c). At this point, the chip is annealed for 1 hour at $1000{ }^{\circ} \mathrm{C}$ to densify and improve the film quality of the oxide and nitride. Following this, we lithographically pattern and dry etch all layers stopping on the Si substrate to define patterns for fiber v-grooves and for chip singulation. Potassium hydroxide $(\mathrm{KOH})$ etching defines $75 \mu \mathrm{m}$ deep v-grooves. During this step, the SOI silicon is sufficiently protected by the BOX and top oxide layers as shown in Fig. 1(d).

We mechanically cleave to singulate $5 \mathrm{~mm} \times 5 \mathrm{~mm}$ die, followed by mechanical polishing of the Si die corner at $45^{\circ}$ from the backside and focused ion beam (FIB) undercut of the transducer area to expose the cantilever probe at the chip corner (Fig. 2) and to sharpen the AFM probe tip. This mechanical processing is needed for unimpeded sample approach with the in-plane fabricated probe in AFM experiments (in the future may be accomplished by an appropriate combination of scalable back and front bulk micromachining techniques). The devices are released by wet etching in $49 \%$ by mass HF solution in water, followed by rinsing and supercritical point drying. Figure 2(a) shows side view of the die corner after mechanical milling for gross under-cut of the $\mathrm{Si}$ substrate under the cantilever with $45^{\circ}$ milling angle using diamond grit sandpaper. Figure 2(b) shows SEM side view image of a finished, FIB-milled and HF-released device. (a)

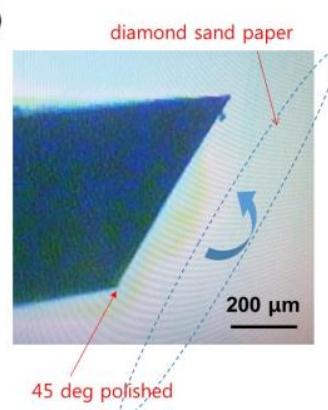

(b)

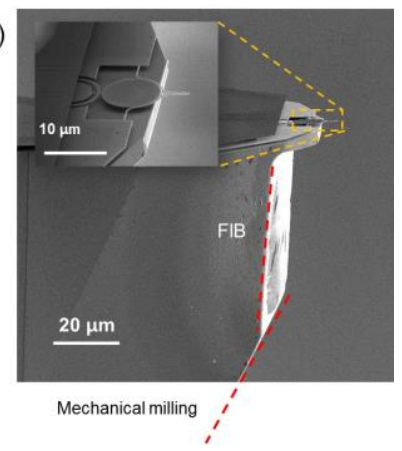

Figure 2: (a) Mechanical milling result. Si substrate under the cantilever is firstly polished by milling machine with diamond sand paper at $45^{\circ}$ angle. (b) FIB milling is precisely performed near the cantilever area. (a)

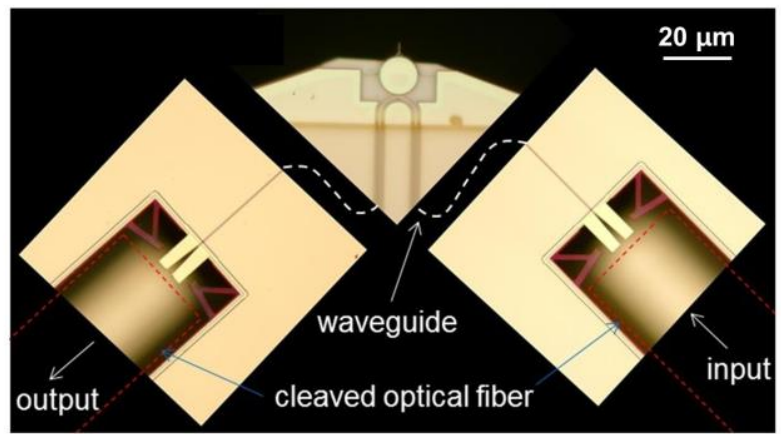

(b)

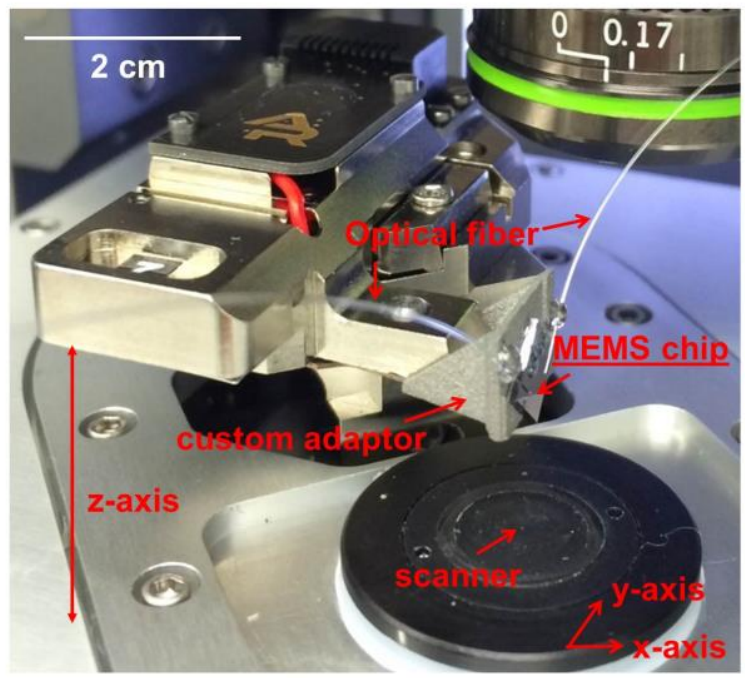

Figure 3: (a) Optical microscope images of the fabricated optomechanical device. (b) Installation of the optical fiber glued device into a commercial AFM system.

Figure 3(a) shows a top down optical microscope view of the optomechanical device and the v-groove terminations with $\mathrm{Si}$ waveguide optical couplers. The released die is attached with epoxy to a custom 3D-printed metal adapter and then bare, cleaved, single mode telecom optical fibers are actively aligned in the v-grooves to the on-chip inverse-taper optical couplers using a $1550 \mathrm{~nm}$ wavelength laser source. The fibers are glued into the v-grooves and to the adapters using ultra-violet (UV) curable epoxy. The fiber facet and the suspended Si inverse taper structure at the end of the v-grooves typically remain epoxy-free.

We install this optical fiber glued device into a commercial AFM system. We only use the chip cantilever chip holder and the sample scanner of the AFM, while our optomechanical signal is fed back into the instrument in place of its normal far-field beam-bounce optical detection system as shown in Fig. 3(b). The active corner on the die with the nanoscale sharpened probe is oriented down toward the sample stage of the AFM.

\section{DEVICE DESIGN AND CHARACTERIZATION}

Figure 4(a) shows an optical micrograph of the fabricated integrated silicon cavity optomechanical transducer with a basic schematic of the AFM measurement. The nominal fabricated disk diameter is $10 \mu \mathrm{m}$, while the cantilever nominal width and length are $120 \mathrm{~nm}$ and $24 \mu \mathrm{m}$, respectively; the gap between cantilever and microdisk is $\approx 200 \mathrm{~nm}$, and the tip radius after FIB sharpening is $\approx 15 \mathrm{~nm}$, which is comparable to the tips of commercial AFM cantilevers. 
Figure 4(b) shows the working principle for a cavity optomechanical transducer-based near-field detection of cantilever motion. The mechanically-stationary microdisk is a high quality factor whispering gallery optical cavity that can be sensed through an evanescently coupled Si waveguide. Coupling into a cavity optical resonance mode results in a spectrally-narrow dip in waveguide transmission, Fig 4b. Optical quality factors of the $10 \mu \mathrm{m}$ diameter microdisk resonator typically lie between 15000 to 100000 depending on the surface cleanliness of the microdisk rim, the roughness of the etched sidewall, and the geometry of the waveguide-microdisk coupling area.

The cantilever is too narrow to support a guided optical mode and to guide any light away from the optical cavity. Thus it does not affect the cavity optical loss. Instead, moving the cantilever into (out of) the evanescent fields of the cavity lowers (increases) the cavity optical resonance frequency, shifting the transmission dip. The shift of the spectrum can be translated into an amplitude modulation of the transmitted light by tuning the excitation laser to a fixed wavelength on the shoulder of the resonance, Fig. 4(b). Therefore, the photodetector voltage becomes linearly proportional to the cantilever position. Figures 5(a) and 7(b) shows that the motion readout noise is low enough to clearly resolve the thermal fluctuations of the mechanical probe itself, both when free and in contact with the sample. Therefore, the measurement performance is determined by the probe's thermal mechanical noise.

The non-contact noise spectrum for the cantilever used in the AFM experiment is shown in Fig. 5(a), measured with $\approx 3 \mathrm{~mW}$ of input optical power. The mechanical stiffness is estimated by finite element calculation to be $\approx 1 \mathrm{~N} / \mathrm{m}$ and cantilever mass is in the sub-picogram range. The observed resonance frequency of $\approx 4 \mathrm{MHz}$ agrees well with the calculation. These parameters together with the measured quality factor $\mathrm{Q} \approx 20$, are used to calibrate the sensitivity using the equipartition theorem. This gives the readout noise background in Fig. 5(a) of $\approx 7 \mathrm{fm} / \mathrm{Hz}^{1 / 2}$ and the thermodynamic

(a)

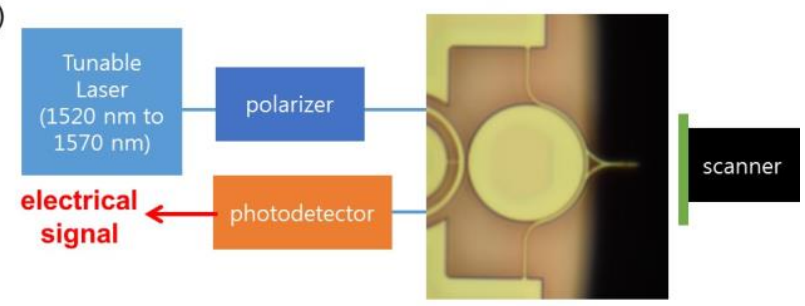

(b)

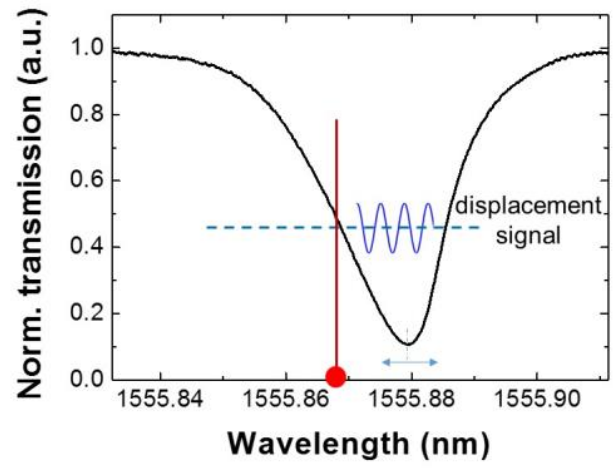

Figure 4: Optomechanical transducer-based nanoscale cantilever. (a) Device and detection schematic. (b) Working principle. (a)

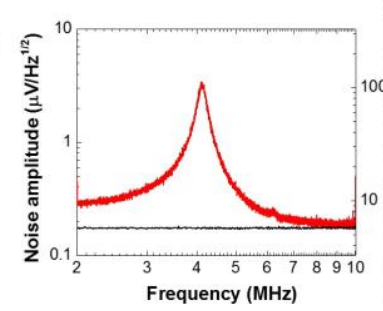

(b)

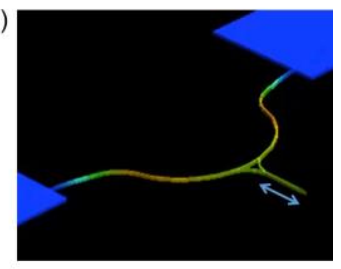

Figure 5: (a) Measured mechanical resonance frequency $(4 \mathrm{MHz})$. (b) Simulation results of corresponding mechanical resonance.

Langevin force noise density of $\approx 6 \mathrm{fN} / \mathrm{Hz}^{1 / 2}$ acting on the cantilever, in air. Note that we can, in principle, widely vary the cantilever resonance frequency $(200 \mathrm{kHz}$ to $110 \mathrm{MHz})$ and stiffness $(0.01 \mathrm{~N} / \mathrm{m}$ to $290 \mathrm{~N} / \mathrm{m})$ by varying the design of e-beam lithography pattern [10].

This motion readout signal can be used by the AFM system as the cantilever "deflection" signal. Following conventional contact mode protocol, the AFM closes a feedback loop by adjusting the height of the probe relative to the sample to maintain deflection near a fixed setpoint value, adjusting for the changes in the sample topography as the probe is scanned.

\section{EXPERIEMTNAL RESULTS}

We performed fast scanning AFM and a PTIR measurement on a single location with the optomechanical transducer-based nanoscale cantilever.

\section{Fast scanning}

Figure 6(a) shows the contact-mode fast-scanning AFM image of a $3 \mu \mathrm{m}$ period grating in ambient conditions. A wide area of 30 $\mu \mathrm{m} \times 30 \mu \mathrm{m}$ is imaged a high resolution $(512 \times 512$ pixels $)$ using a line scan rate of $39.06 \mathrm{~Hz}$ in the fast direction, which corresponds to a cantilever linear speed of $2.93 \mathrm{~mm} / \mathrm{s}$ (total scan time: $13.5 \mathrm{~s}$ ). Note that these times and frequencies properly account for the additional scanner turn-around time between each line. Even at this speed, the cantilever followed the sharp $25 \mathrm{~nm}$ high steps of the surface topography of the grating as shown in the Topo + Deflection images
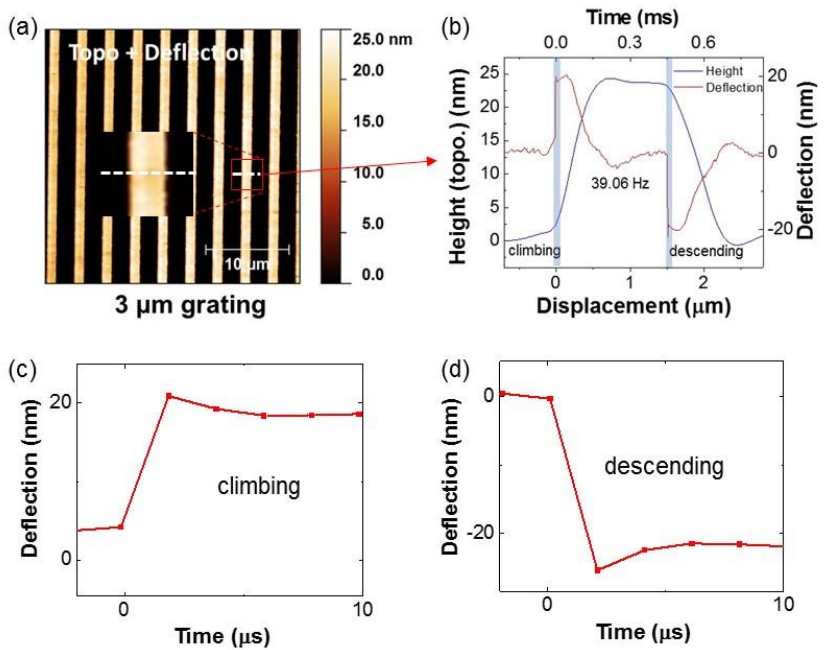

Figure 6: AFM measurement of a test grating. (a) AFM image of the $25 \mathrm{~nm}$ tall grating lines. (b)-(d) a single line scan. Experimental uncertainty is smaller than the data marker size. 
(a)
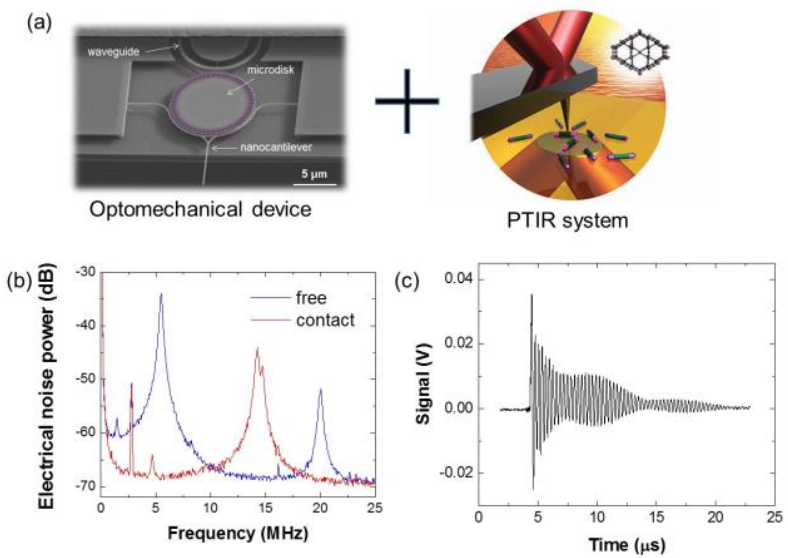

Figure 7: PTIR measurement. (a) Optomechanical probe is used instead of a conventional AFM cantilever and its beam-bounce readout in PTIR nanoscale chemically-sensitive imaging system. (b) Cantilever probe is limited by fundamental thermodynamic mechanical noise which is lower than thermal noise of conventional cantilevers in air. Noise power is relative to $1 \mathrm{~mW}$. (c) Signal from a $50 \mathrm{~nm}$ thick PMMA film.

and line profiles in Fig. 6(b). The cantilever deflection changes very quickly at the grating steps, followed by the slower compensation by the AFM z feedback ("topography" channel). At the steps, the cantilever climbs up the wall (Fig. 6(c)) and descends the valley (Fig. 6(d)) with the response that is within about $20 \%$ of the final value achieved within the $2 \mu$ s single time step duration. Based on the standard deviation among 10 adjacent points on a flat portion of the sample, taken at $2 \mu$ s per point, the noise per point is estimated as $20 \mathrm{pm}$ one standard deviation.

\section{PTIR measurement}

We have integrated our optomechanical probe into a commercial photo-thermal induced resonance (PTIR) system [11]. PTIR combines the nanoscale resolution of AFM and optical spectroscopy and enables measuring local infrared absorption spectra and mapping chemical composition with nanoscale resolution $[12,13]$. The system operates by illuminating a thin-film sample with a short pulse from a tunable infrared laser. If the sample at a given location absorbs the specific infrared wavelength of the pulse, the optical energy is locally converted into heat and results in surface motion due to thermal expansion. An AFM cantilever in contact with the sample at such location experiences a mechanical "kick" which is detected. Typically, the short thermo-mechanical kick from the sample excites one or more mechanical resonant modes of the cantilever, and amplitude of the cantilever vibration ringdown provides a measure of local infrared absorption.

The exquisitely high-precision motion readout and low thermal noise of our nanoscale sensor compared to larger cantilevers in air will enable fast PTIR measurements of extremely thin samples. The low readout noise means fewer ringdown traces have to be averaged to achieve good signal-to-noise ratio at each point, and therefore faster acquisition of images and absorption spectra will be possible. By substituting our nanoscale cantilever and optical readout instead of a conventional microscale cantilever and beam-bounce readout (Fig. 7(a)), and leveraging the smaller thermomechanical and readout noises of our system (Fig. 7b), in preliminary experiments we were able to easily measure the PTIR ringdown signals from a test film of poly (methyl methacrylate) (PMMA) only about $50 \mathrm{~nm}$ thick (Fig. 7c) with 1024 averages.

\section{CONCLUSION}

We have integrated an optomechanical transducer-based nanoscale cantilever into AFM and PTIR systems. We demonstrate fully-functional contact mode AFM imaging with the probe, highlighting fast scanning and low noise. We further integrate the probe in a photo-thermal induced resonance system, and detect PTIR signal from a thin sample.

\section{REFERENCES}

[1] N. Kodera, D. Yamamoto, R. Ishikawa, and T. Ando, "Video imaging of walking myosin $\mathrm{V}$ by high-speed atomic force microscopy", Nature, 468, 72-76 (2010).

[2] M. Shibata, H. Yamashita, T. Uchihashi, H. Kandori, and T. Ando, "High-speed atomic force microscopy shows dynamic molecular processes in photoactivated bacteriorhodopsin", Nat. Nanotech. 5, 208-212 (2010).

[3] T. Uchihashi, R. Iino, T. Ando, and H. Noji, "High-Speed Atomic Force Microscopy Reveals Rotary Catalysis of Rotorless F1-ATPase", Science, 333, 755-758 (2011).

[4] K. Srinivasan, H. Miao, M. T. Rakher, M. Davanço, and V. Aksyuk, "Optomechanical Transduction of an Integrated Silicon Cantilever Probe Using a Microdisk Resonator", Nano Lett., 11(2), 791-797 (2011).

[5] D. Woolf, P.-C. Hui, E. Iwase, M. Khan, A. W. Rodriguez, P. Deotare, I. Bulu, S. G. Johnson, F. Capasso, and M. Loncar, " Optomechanical and photothermal interactions in suspended photonic crystal membranes", Opt. Exp. 21(6), 7258-7275 (2013).

[6] S. L. Portalupi, M. Galli, C. Reardon, T. F. Krauss, L. O'Faolain, L. C. Andreani, and D. Gerace, "Planar photonic crystal cavities with far-field optimization for high coupling efficiency and quality factor", Opt. Exp. 18, 16064 (2010).

[7] K. Srinivasan and O. Painter, "Linear and nonlinear optical spectroscopy of a strongly coupled microdisk-quantum dot system", Nature 450, 862-865 (2007).

[8] M. Eichenfield, C. P. Michael, R. Perahia, and O. Painter, "Actuation of micro-optomechanical systems via cavity-enhanced optical dipole forces", Nat. Photon. 1, 416-422 (2007).

[9] F. Roozeboom, F. van den Bruele, Y. Creyghton, P. Poodt, and W. M. M. Kessels, "Cyclic Etch/Passivation-Deposition as an All-Spatial Concept toward High-Rate Room Temperature Atomic Layer Etching", ECS J. Solid State Sci. Technol. 4, N5067-N5076 (2015).

[10] Y. Liu, H. Miao, V. Aksyuk, and K. Srinivasan, "Wide cantilever stiffness range cavity optomechanical sensors for atomic force microscopy", Optics Exp. 20, 18268-18280 (2012).

[11] A. M. Katzenmeyer, V. Aksyuk, and A. Centrone, "Nanoscale Infrared Spectroscopy: Improving the Spectral Range of the Photothermal Induced Resonance Technique", Anal. Chem., 85 (4), pp 1972-1979 (2013).

[12] B. Lahiri, G. Holland, V. Aksyuk, and A. Centrone, "Nanoscale Imaging of Plasmonic Hot Spots and Dark Modes with the Photothermal-Induced Resonance Technique", Nano Lett., 13, 3218-3224 (2013).

[13] A. M. Katzenmeyer, J. Canivet, G. Holland, D. Farrusseng, and A. Centrone, "Synthetic Assessing chemical heterogeneity at the nanoscale in mixed-ligand metal-organic frameworks with the PTIR technique", Angew. Chem. Int. Ed., 53, 2852-2856 (2014).

\section{CONTACT}

*V. Aksyuk, tel: +1-301-975-2867; vladimir.aksyuk@nist.gov 